\title{
Internato médico rural na Serra Gaúcha: a experiência da Universidade de Caxias do Sul
}

\author{
Rural medical internship in Serra Gaúcha: the experience \\ of the Universidade de Caxias do Sul
}

\author{
Leonardo Vieira Targa ${ }^{1}$, Naiane Dartora dos Santos ${ }^{2}$, Fábio Schwalm³, \\ Enrique Falceto de Barros ${ }^{4}$, Vanessa Laubert La Porta ${ }^{2}$, Guilherme \\ Toscani $^{5}$, Asdrubal Falavigna ${ }^{6}$, Tatiana Souza de Camargo ${ }^{7}$
}

Resumo

Um dos desafios das comunidades rurais e remotas é a dificuldade no recrutamento e a retenção de recursos humanos em saúde. A educação médica em áreas rurais é uma das estratégias para enfrentar tal deficiência. No Brasil, há relativamente poucos relatos de experiências de educação médica em áreas rurais, especialmente de duração prolongada, e há necessidade de se avaliar as existentes para contribuir com a formulação de políticas públicas. Este trabalho descreve o estágio rural do internato médico em Medicina de Família e Comunidade (MFC) da Universidade de Caxias do Sul (UCS). Até agora, durante os treze anos desde seu início, mais de 550 estagiários passaram pelas unidades de saúde de quatro municípios da Serra Gaúcha. Reflexões sobre a experiência para a formação médica e para as políticas de recursos humanos são apresentadas. O estágio rural tem sido importante espaço de diversificação de cenário para educação, reforçando o papel da Atenção Primária à Saúde e da MFC, além de possibilitar a aproximação da Universidade com a comunidade, confirmando sua responsabilidade social. Os resultados contribuem para as estratégias de formação médica e para as políticas de recrutamento e retenção de recursos humanos na saúde para áreas rurais e remotas.

Palavras-chave: Educação médica. Atenção Primária à Saúde. Medicina de família e comunidade. Áreas rurais. Recursos humanos em saúde.

\footnotetext{
${ }^{1}$ Doutorando em Educação em Ciências: Química da Vida e Saúde na Universidade Federal do Rio Grande do Sul (UFRGS), Porto Alegre, Rio Grande do Sul, Brasil. Professor do Curso de Graduação em Medicina na Universidade de Caxias do Sul (UCS), Caxias do Sul, Rio Grande do Sul, Brasil. E-mail: lvtarga@ucs.br

${ }^{2}$ Residência Médica em Medicina de Família e Comunidade pelo Hospital Nossa Senhora da Conceição de Porto Alegre, Porto Alegre, Rio Grande do Sul, Brasil. Professoras do Curso de Graduação em Medicina na Universidade de Caxias do Sul, Caxias do Sul, Rio Grande do Sul, Brasil.

${ }^{3}$ Mestrando no Programa de Pós-graduação em Avaliação de Tecnologias para o SUS - GHC do Grupo Hospitalar Conceição, Porto Alegre, Rio Grande do Sul, Brasil.

${ }^{4}$ Mestrando no Programa de Pós-graduação em Educação em Ciências: Química da Vida e Saúde da Universidade Federal do Rio Grande do Sul, Porto Alegre, Rio Grande do Sul, Brasil. Professor do Curso de Graduação em Medicina na Universidade de Caxias do Sul, Caxias do Sul, Rio Grande do Sul, Brasil.

${ }^{5}$ Mestrado em Ciências Biológicas (Fisiologia) pela Universidade Federal do Rio Grande do Sul, Porto Alegre, Rio Grande do Sul, Brasil. Médico de Família na Unidade de Estratégia Saúde da Família (ESF) Zebert Kraupp e Clínico Geral no Ambulatório Geral Mário Jorge Vieira, Blumenau, Santa Catarina, Brasil.

${ }^{6}$ Doutorado em Neurociências pela Universidade Federal de São Paulo (Unifesp), São Paulo, São Paulo, Brasil. Professor do Curso de Graduação em Medicina na Universidade de Caxias do Sul, Caxias do Sul, Rio Grande do Sul, Brasil.

${ }^{7}$ Doutorado em Educação pela Universidade Federal do Rio Grande do Sul, Porto Alegre, Rio Grande do Sul, Brasil. Professora do Departamento de Ensino e Currículo da Faculdade de Educação da Universidade Federal do Rio Grande do Sul, Porto Alegre, Rio Grande do Sul, Brasil.
} 


\begin{abstract}
One of the challenges of rural and remote communities is the difficulty in recruiting and retaining human resources in health. Medical education in rural areas is one of the strategies to tackle this deficiency. In Brazil, there are relatively few reports of medical education experiences in rural areas, especially of long duration, and there is a need to evaluate the existing ones to generate useful reflections for the formulation of public policies. This work describes the medical internship in Family Medicine (FM) at the Universidade de Caxias do Sul (UCS). To date, during the thirteen years since its inception, more than 550 medical students have passed through four municipalities in the Serra Gaúcha. Reflections on the experience about medical training and human resources policies for rural health are presented. The rural internship has been an important space for diversifying the scenario for medical education, reinforcing the role of Primary Health Care and of FM, in addition to making it possible to bring the University closer to the community, confirming its social responsibility. The results of this report contribute to medical training strategies and policies for the recruitment and retention of human resources in health for rural and remote areas.
\end{abstract}

Keywords: Medical education. Primary Health Care. Family medicine. Rural areas. Human resources in health.

\section{Introdução}

A falta de profissionais de saúde em zonas rurais e remotas e as dificuldades de acesso aos recursos de saúde estão entre os principais obstáculos para melhorar a saúde nas áreas rurais e para uma maior equidade entre a saúde rural e a urbana. A Organização Mundial da Saúde (OMS) refere que, enquanto aproximadamente metade da população do globo habita zonas rurais, menos de um quarto dos médicos trabalha nestas áreas. ${ }^{(1)}$ A falta de profissionais de saúde chega a 10 milhões no mundo e este déficit é distribuído desigualmente, ocorrendo perto de $70 \%$ nas áreas rurais. ${ }^{(2)}$ Esse problema é ainda mais sério nos países menos desenvolvidos, ${ }^{(3)}$ embora não seja exclusivo destes.

Os fatores citados na literatura, relacionados a esse problema, bem como as estratégias testadas para enfrentá-lo são: 1) os relacionados à seleção dos estudantes (formas de ingresso, origem geográfica, características de personalidade, gênero e renda); 2) os relacionados com a própria formação (localização do curso, modelos de rotação de estágios, exposição a diferentes cenários de ensino, direcionamento dos currículos para as necessidades locais, programas compulsórios); e 3) pós-graduação (estímulos de recrutamento e retenção baseados nos fatores anteriormente citados, análise de desempenho em provas, pós-graduação rural, educação permanente, fortalecimento das chances de ligação do profissional rural com carreira acadêmica e pesquisa, características da docência rural, suporte e incentivos). ${ }^{(4)}$ A OMS recomenda abordagens múltiplas às nações que enfrentam dificuldades no recrutamento e retenção de profissionais de saúde para áreas rurais. Dentro dessa abordagem múltipla, são citados os fatores relacionados à formação profissional como importantes indutores de uma melhor distribuição posterior dos profissionais. ${ }^{(1)}$ Evidências internacionais acumulam-se reforçando esta recomendação. ${ }^{(4-6)}$

A educação médica em áreas rurais ou cidades de pequeno porte apresenta outras vantagens para o profissional em formação, para os serviços de saúde locais e para as comunidades. Entre elas estão: o conhecimento de realidades diferentes dos grandes centros urbanos, onde a maioria das faculdades se concentra; aprimoramento de habilidades de comunicação; treinamento de competência intercultural; ampliação do espectro de habilidades exigidas aos médicos em regiões onde recursos especializados e interdisciplinares são escassos; percepção de diferentes formas de cooperar em equipe; treinamento da aplicação do conhecimento médico em ambientes diferentes; promoção da resiliência; aprendizado através do feedback de uma comunidade; treinamento de aspectos coletivos/comunitários do cuidado à saúde de populações delimitadas. ${ }^{(7-10)}$ 
A formação médica vem passando por transformações, especialmente nas últimas duas décadas. Entre essas transformações estão uma maior ênfase na Atenção Primária à Saúde (APS), buscando uma formação generalista e uma aproximação do meio acadêmico com as necessidades das pessoas, inseridas em seu contexto social. ${ }^{(11)}$ As experiências de interiorização na formação médica são parte importante, embora ainda estejam longe de ser comuns nos currículos médicos brasileiros.

Desde 2013, o Brasil conta com a Política Nacional de Saúde Integral das Populações do Campo e da Floresta (PNSIPCF). ${ }^{(12)}$ Esta política induz a consideração dos temas relacionados às necessidades da saúde rural na educação permanente dos profissionais, o que pode e deve evidentemente ser extrapolado para sua formação inicial.

Apesar disso, ainda são relativamente escassos os relatos com maior profundidade de experiências de educação médica em áreas rurais no País. Há grande necessidade de se avaliar as existentes, bem como aproveitar sua história para reflexão que auxilie na construção de políticas de promoção da saúde das populações rurais. Este relato tem como objetivo contribuir para a diminuição dessa lacuna.

\section{Material e Método}

Este trabalho busca realizar um relato e reflexões a partir de uma experiência de educação médica em área rural. A experiência destacada é a do estágio rural em Saúde da Família da Universidade de Caxias do Sul (UCS), estado do Rio Grande do Sul. É um estudo retrospectivo da história desse estágio a partir de dados oriundos dos registros referentes aos alunos e atividades por eles desenvolvidas e a recordação pessoal dos professores médicos de família e comunidade envolvidos com o mesmo e dos coordenadores do internato médico em Medicina de Família e Comunidade (MFC) e da coordenação de curso à época do seu início. Os dados são apresentados de forma qualitativa e quantitativa, e buscam descrever a evolução e os diferentes momentos do estágio dentro do curso médico. Os pontos destacados estão relacionados aos seguintes aspectos: etapas para criação do estágio; momentos importantes de sua evolução posterior; características dos locais de estágio; critérios de adesão; tempo e duração; formas de organização e descrição das principais atividades realizadas pelos estagiários; número de estagiários; descrição de produtos derivados da experiência educacional; e os principais desafios encontrados. Reflexões a partir da experiência dos autores também são realizadas e elencadas implicações para o sistema de saúde, sistema educacional médico e para a saúde da comunidade. Este trabalho não envolve diretamente intervenção em seres humanos, não havendo, portanto, necessidade de aprovação em comitê de ética.

\section{Resultados}

\section{Breve histórico}

O primeiro passo para a construção do estágio rural ocorreu em 2007, quando estudantes do curso de Medicina da UCS solicitaram a realização de estágio voluntário de férias em Nova Petrópolis (RS), na Unidade de Saúde Pinhal Alto (Figura 1). $\mathrm{O}$ interesse das estudantes foi despertado nas aulas de APS, na qual o professor - que é médico naquele município - utiliza exemplos e comparações provenientes da sua experiência em áreas rurais.

Figura 1 - Foto tirada em frente ao posto de saúde por um estagiário; primeiros momentos do estágio.

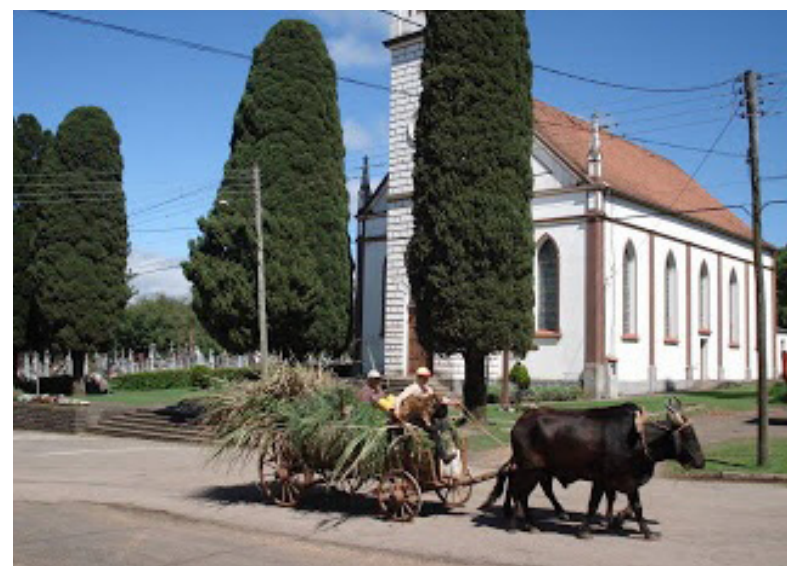

Fonte: Estagiário do projeto 
No mesmo ano, por iniciativa de três outras alunas, houve uma atividade educacional sobre a relação entre saúde e hábitos de vida, na Escola
Estadual São José, município de Nova Petrópolis, Rio Grande do Sul, na área de abrangência da mesma unidade de saúde (Figura 2).

Figura 2 - Atividade educativa na escola São José, no interior de Nova Petrópolis.

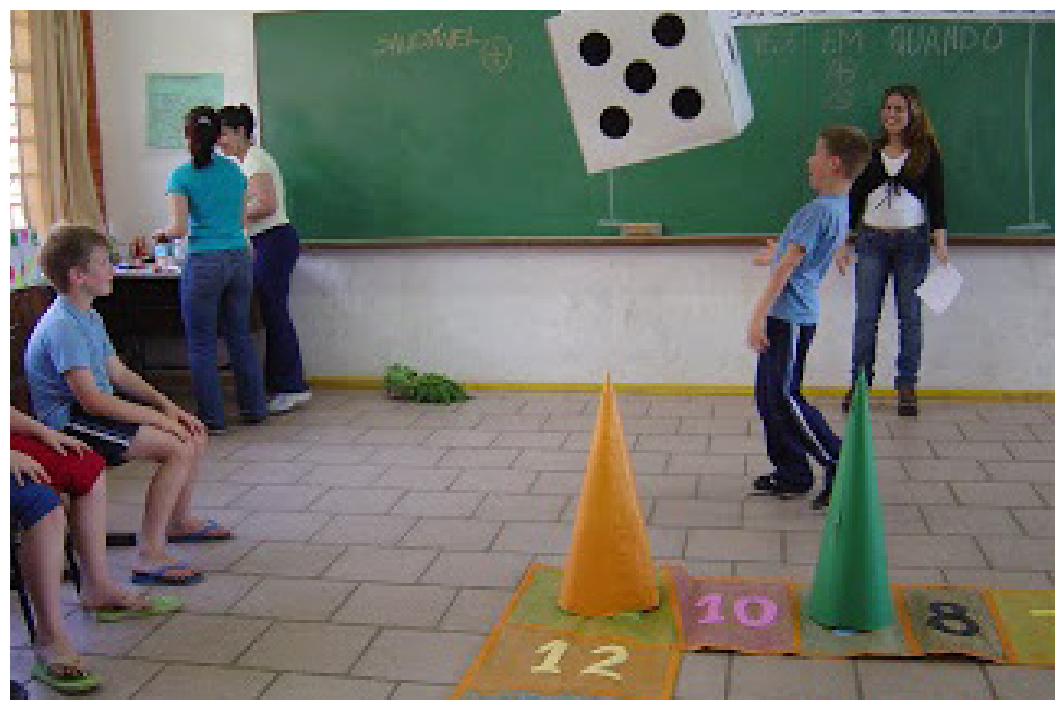

Fonte: Autores

A partir destas experiências exitosas, foram buscadas novas parcerias com instituições de ensino. Uma feira de saúde foi montada em parceria com a Universidade Federal de Ciências da Saúde de Porto Alegre (UFCSPA). Em 2008, foi realizada uma visita técnica com preceptores e residentes médicos do Programa de Residência Médica em Medicina de Família e Comunidade do Grupo Hospitalar Conceição (GHC), sendo acordado que a Unidade de Saúde do Pinhal Alto seria um local oferecido aos residentes para estágios voluntários. A Secretaria de Saúde e Assistência Social de Nova Petrópolis, oficializou em seguida seu apoio à vinda dos médicos residentes, especialmente através de um acordo de estadia econômica com um albergue local. Este acordo formalizou-se posteriormente em um convênio de cooperação entre as instituições que contribuiu para o amadurecimento de uma relação mais forte entre ensino e serviço.

$\mathrm{O}$ interesse dos alunos de graduação em Medicina da UCS foi tornando-se maior conforme alguns estagiários voluntários iam participando de forma intercalada ou concomitante com os residentes anteriormente descritos. Estas experiências apresentavam avaliação positiva tanto por parte dos alunos, quanto da equipe e comunidade envolvida. Estas avaliações transpuseram o aspecto pontual e chegaram à coordenação do curso de Medicina da Universidade. Estes acontecimentos somaramse ao momento propício, em que havia tanto uma coordenação de curso sensível às necessidades de formação médica e assistências às comunidades rurais, quanto políticas de educação médica que sofreram reformulações valorizando a Atenção Primária à Saúde e a inserção comunitária.

Em 2011, oficializou-se a parceria entre UCS e o município de Nova Petrópolis, a partir de um convênio entre as instituições, gerando um fluxo contínuo de estudantes dos últimos dois anos do curso de graduação em Medicina (internos) e formalizando apoio financeiro da Universidade para os estagiários.

Progressivamente, esta parceria foi substituindo os estágios de residentes, por questões de espaço físico e qualidade, apesar de estes nunca deixaram totalmente de ocorrer. Outros médicos de família e comunidade de áreas rurais e pequenos municípios próximos foram sendo incorporados 
no grupo de professores de Atenção Primária à Saúde na UCS. Estes também eram procurados para receber residentes e isso acabou por fortalecer o caminho de naturalmente tornarem-se locais oficiais de estágio também para os estudantes da Universidade de Caxias do Sul.

A partir de janeiro de 2015, o estágio foi ampliado para estes municípios, inicialmente Barão (RS) e Santa Maria do Herval (RS) e, após 2018, São Pedro da Serra (RS). Isso gerou um importante aumento das oportunidades para os estagiários e uma maior variedade de situações de aprendizado.

O estágio

O internato rural em Saúde da Família da UCS é uma oportunidade de formação médica em nível de graduação, de caráter voluntário. Pretende oferecer uma vivência em medicina no Sistema Único de Saúde (SUS) em cenário de relativo isolamento do restante do sistema e em equipes menores. Ele integra o internato de Medicina de Família e Comunidade, que é uma das seis áreas obrigatórias de estágio curricular para a graduação em Medicina na UCS. O estágio na área de Medicina de Família e Comunidade tem uma duração de cerca de 95 dias. Atualmente dispõe de dez vagas mensais em internato rural, visto que os alunos são alocados em duplas ou trios em cada localidade por período aproximado de 30 dias.

O estagiário acompanha e participa ativamente de todas as atividades de um médico de uma Unidade de Saúde da Família (USF) em área rural, que também é professor especialista em medicina de família e comunidade (MFC). Estas atividades incluem consultas médicas sob supervisão, visitas domiciliares (Figura 3), procedimentos cirúrgicos ambulatoriais (Figura 4), atividades de educação em saúde (Figura 5), planejamento e territorialização, e reuniões de equipe e da secretaria de saúde.

Estimula-se o estudo baseado em evidências e a aplicação culturalmente competente dos principais problemas de saúde que acometem as populações rurais do Sul do Brasil. O tempo de estágio é, em média, de 4 semanas (de 3-6 semanas) e a carga horária é de segundas às quintas-feiras nos turnos da manhã e tarde; de acordo com o estabelecido na Lei de Estágio vigente, respeita-se as 30 horas de atividade semanal. Atividades em outros horários e dias eventualmente são combinadas de acordo com o interesse e disponibilidade do estudante e podem incluir visitas a outras unidades de saúde do município, hospital, secretaria de saúde e Centros de Atenção Psicossocial (CAPS), além de atividades de pesquisa, participação em atividades relacionadas ao controle social e educação em saúde.

Figura 3 - Visita domiciliar em Santa Maria do Herval.

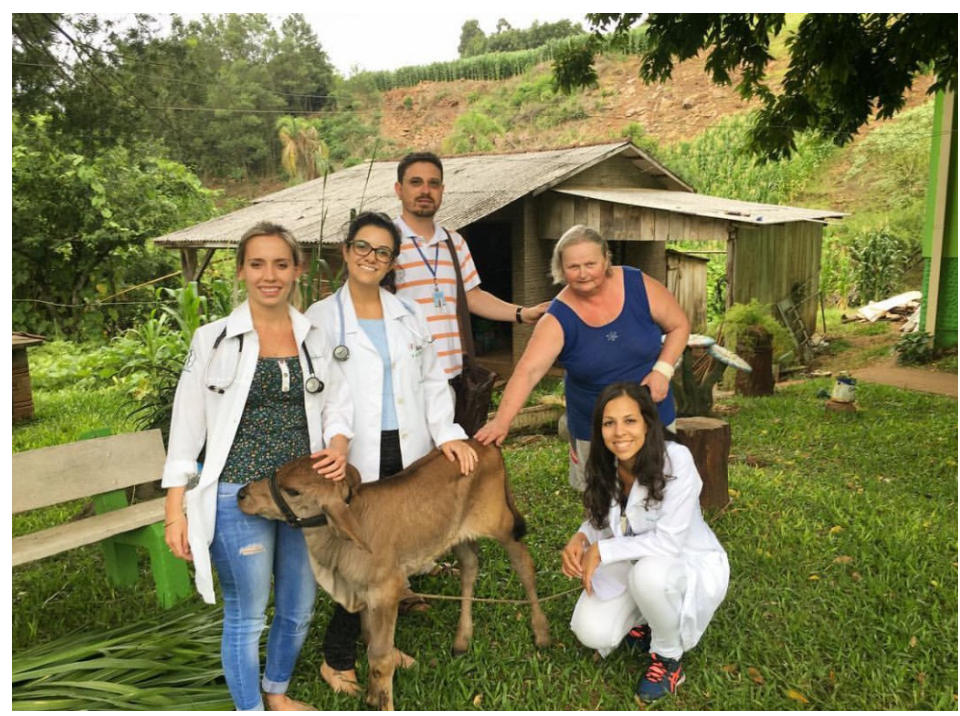

Fonte: Autores 
As regiões onde ocorre o estágio apresentam características próprias, embora semelhantes. As populações adscritas, as distâncias dos centros dos municípios e as distâncias dos pequenos hospitais do interior, bem como dos serviços de mais alta complexidade, e também as datas de início dos estágios e número de estagiários, podem ser visualizados no Quadro 1.

Quadro 1 - Algumas características dos locais de estágio.

\begin{tabular}{|c|c|c|c|c|c|c|}
\hline Local & $\begin{array}{c}\text { População } \\
\text { adscrita }\end{array}$ & $\begin{array}{c}\text { Distância } \\
\text { do centro } \\
\text { do município }\end{array}$ & $\begin{array}{c}\text { Distância } \\
\text { de hospital } \\
\text { pequeno porte }\end{array}$ & $\begin{array}{c}\text { Distância de } \\
\text { referência alta } \\
\text { complexidade }\end{array}$ & $\begin{array}{c}\text { Total de } \\
\text { Ano de início } \\
\text { atividade } \\
\text { estagiários } \\
\text { recebidos } \\
\text { (não só UCS, } \\
\text { até 3/2020) }\end{array}$ \\
\hline $\begin{array}{c}\text { Nova Petrópolis } \\
\text { (Pinhal Alto) }\end{array}$ & 2162 & $15 \mathrm{~km}$ & $15 \mathrm{~km}$ & $50 \mathrm{~km}$ & 2007 & 233 \\
\hline $\begin{array}{c}\text { Santa Maria } \\
\text { do Herval }\end{array}$ & 3500 & $0 \mathrm{~km}$ & $22 \mathrm{~km}$ & $79 \mathrm{~km}$ & 2015 & 130 \\
\hline $\begin{array}{c}\text { Barão } \\
\text { (Francesa Alta) }\end{array}$ & 1900 & $10 \mathrm{~km}$ & $10 \mathrm{~km}$ & $38 \mathrm{~km}$ & 2015 & 132 \\
\hline Barão (Centro) & 3800 & $0 \mathrm{~km}$ & $50 \mathrm{~m}$ & $35 \mathrm{~km}$ & 2018 & 27 \\
\hline $\begin{array}{c}\text { São Pedro } \\
\text { da Serra }\end{array}$ & 3500 & $0 \mathrm{~km}$ & $3 \mathrm{~km}$ & $45 \mathrm{~km}$ & 2018 & 32 \\
\hline
\end{tabular}

Fonte: Autores

Figura 4 - Procedimento cirúrgico ambulatorial de urgência em Barão.

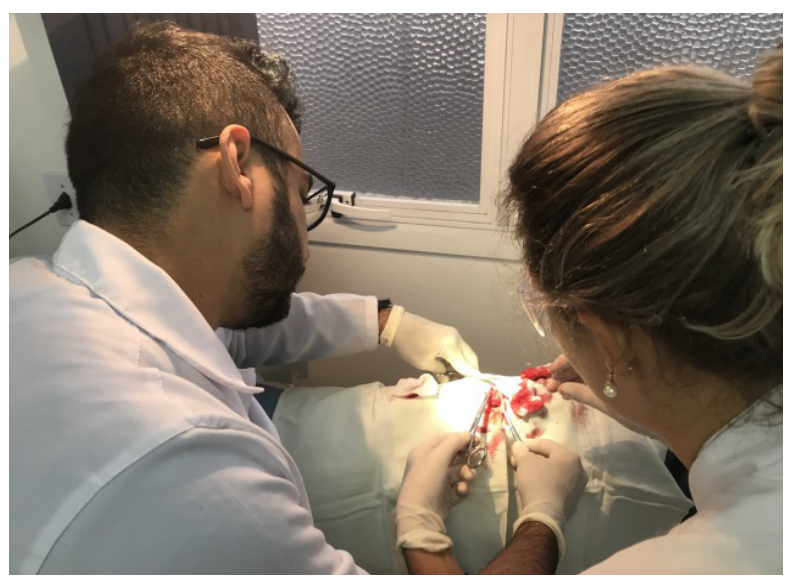

Fonte: Autores

Além das atividades do estágio rural, os internos mantêm a participação das atividades do internato de Medicina de Família e Comunidade, que ocorrem em Caxias do Sul, na Universidade de Caxias do Sul, nas quintas-feiras à tarde e sextasfeiras durante o dia. Estas atividades incluem aulas teóricas de temas relevantes à área, revisão de encaminhamentos para especialistas de unidades específicas do município e quinzenalmente realizase uma atividade em grupo, o momento Balint. $\mathrm{O}$ grupo Balint ${ }^{(13)}$ tem como objetivo o aprimoramento da relação profissional médico-paciente. No contexto de alunos de graduação, o método é adaptado para trabalhar os sentimentos resultantes dos atendimentos e as dificuldades enfrentadas pelos alunos durante o internato. Observamos que esse encontro é de grande valia, uma vez que é dada oportunidade aos alunos para falarem sobre suas experiências e melhorar a forma de abordagem com os pacientes nos futuros atendimentos.

Até o mês de março de 2020, 558 estagiários tiveram parte de sua formação nas diversas localidades listadas no Quadro 1. Alguns residentes e estudantes de diversas regiões do Brasil e de outros países (Portugal, Espanha, França, Alemanha e Tailândia) também passaram pela experiência, por meio de acordos realizados diretamente com os professores e municípios, e estão incluídos no número acima. 
Uma avaliação com os primeiros 18 residentes de medicina de família que estagiaram em Nova Petrópolis mostrou resultados concordantes com a literatura internacional, sugerindo um aumento da probabilidade de trabalho em áreas rurais por profissionais que tiveram parte de sua formação nestas áreas. ${ }^{(14)}$ Atualmente, após um acúmulo considerável de experiência, está em andamento uma avaliação quali-quantitativa com foco nos aspectos educativos.

Figura 5 - Atividade educativa para funcionárias da creche em Nova Petrópolis.

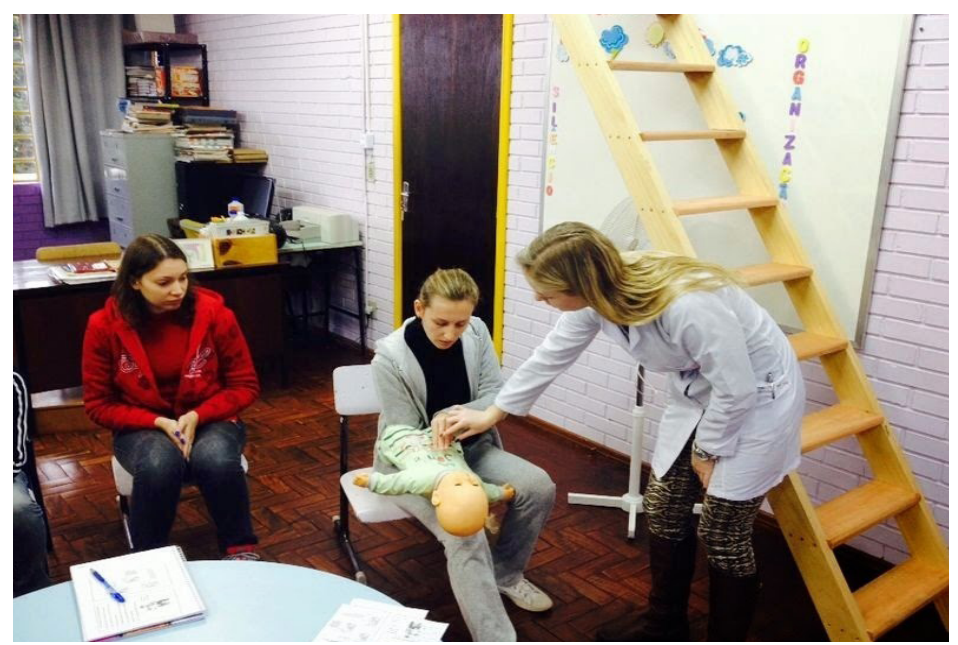

Fonte: Autores

A experiência do estágio rural tem sido divulgada nos últimos anos, nacional e internacionalmente, tanto em congressos médicos quanto de educação médica. Um ponto de destaque ocorreu durante o Congresso Mundial de Saúde Rural, da Organização Mundial de Médicos de Família (WONCA), em 2014. Este importante evento foi trazido para a Serra Gaúcha, para a primeira edição na América Latina, por importante protagonismo dos professores e pelo apoio da Universidade de Caxias do Sul. Na ocasião, as unidades de saúde de Nova Petrópolis e Santa Maria do Herval, que são campos de estágio rural, receberam visitantes internacionais, como atividade pós-congresso, com objetivo de conhecer o Sistema Único de Saúde, a medicina de família brasileira e a experiência local de ensino rural.

\section{Discussão}

Parece inegável que ocorreram alguns avanços na última década em nosso País, em relação à saúde rural. Isto pode ser constatado, por exemplo, a partir da Política Nacional de Saúde Integral das Populações do Campo e da Floresta ${ }^{(12)}$ e das políticas de interiorização de profissionais de saúde, como o Programa Mais Médicos (PMM), ${ }^{(15)}$ no que pese os problemas que podem ser apontados nestas políticas. Além disso, há avanços na forma de pensar o próprio conceito de ruralidade, que pode ser visualizado na evolução do mesmo pelo Instituto Brasileiro de Geografia e Estatística (IBGE), ${ }^{(16)}$ que vai na contramão histórica de minimizar e desvalorizar a ruralidade como residual do processo de urbanização e portanto menos importante. Estes progressos vêm acompanhados de uma tendência a se valorizar o papel da Atenção Básica na formação de profissionais de saúde ${ }^{(11)}$ como foi citado anteriormente. Esta combinação tem sido propícia para repensar os cenários e práticas de educação médica, inclusive promovendo ampliação do uso de espaços rurais.

Estas reconfigurações têm raízes em experiências prévias em áreas rurais. Silveira e Pinheiro $^{(17)}$ nos ajudam a entender uma pouco deste percurso histórico de ações médicas e de 
educação médica em áreas rurais. As experiências mais antigas do gênero em nosso País parecem ser as do internato rural da Universidade Federal de Alagoas (UFAL) (1973) $)^{(9)}$ e da Universidade Federal de Minas Gerais (UFMG) (1978). ${ }^{(18-19)}$ Além disso, existe todo um caminho de políticas de interiorização médica que esteve mais ou menos relacionada à educação, como o Projeto Rondon, o Programa de Interiorização das Ações de Saúde e Saneamento (PIASS), o Programa de Interiorização do Trabalho em Saúde (PITS), o Programa de Valorização da Atenção Básica (PROVAB) e, mais recentemente, o Programa Mais Médicos (PMM) e o Programa Médicos pelo Brasil (PMPB).

Várias experiências em regiões diferentes do Brasil têm implantado modelos variados de ações rurais na formação médica. ${ }^{(9-10,18-21)}$ A partir da análise desta literatura, alguns obstáculos podem ser identificados. O primeiro diz respeito à heterogeneidade e falta de articulação entre as experiências, que na maior parte do tempo parecem depender mais de iniciativas individuais e/ou locais do que de uma ação sistemática governamental que estimule, oriente e organize as ações. Isso evidentemente prejudica a continuidade das experiências. Muitos dos relatos existentes são apenas comunicações resumidas em anais de congressos, o que dificulta o aprofundamento das discussões e provavelmente está relacionado à relativa desarticulação com a pesquisa destas experiências, embora algumas dissertações e teses também estejam disponíveis. ${ }^{(9,18,22)}$

Uma sistematização do conhecimento produzido até aqui se faz necessária, com intuito de contribuir para a formação das políticas na área, papel que deve ser atribuído também às universidades. ${ }^{(10)}$ A própria Política Nacional de Saúde Integral das Populações do Campo e da Floresta (PNSIPCF) não aprofunda a questão da temática rural inserida na formação, embora cite a educação permanente e a educação em saúde como espaços estratégicos. A análise dos desafios e lições aprendidas nesta experiência, relatadas a seguir, se propõe a contribuir para uma redução destas lacunas.

\section{Desafios e lições aprendidas}

Alguns aspectos podem ser destacados como pontos positivos da experiência: o apoio da Universidade foi essencial para a consolidação do projeto, sem este apoio, provavelmente ficar-se-ia restrito a estágios isolados e informais de férias, que normalmente só atingem os alunos altamente motivados e que já pensam em trabalhar no interior. Sem esse apoio, provavelmente não haveria a expansão para todos os centros, visto que alguns colegas professores iniciaram na Universidade também motivados pela possibilidade de receber alunos durante a semana.

$\mathrm{O}$ fato de os professores que recebem alunos terem formação em MFC e serem os médicos das unidades básicas de saúde (UBSs) é um importante diferencial de outras experiências similares. ${ }^{(23)} \mathrm{O}$ conhecimento da comunidade, da epidemiologia e cultura locais, com longitudinalidade (há professores há mais de 15 anos na mesma localidade) aparece sempre nas avaliações dos estudantes como experiência importante para a formação médica, ainda mais por ser rara ao longo do curso. A formação especializada ainda contribui para a melhor demonstração dos princípios da APS e das características únicas do atendimento generalista, centrado na pessoa, família e comunidade, aprimorando o já demonstrado de forma geral para o internato rural por Guimarães. ${ }^{(18)}$ Estes profissionais também têm áreas de interesse especiais diversas, cuja inserção acadêmica tem potencializado, e que são diferenciais interessantes aos alunos como a relação entre Saúde e Espiritualidade, Saúde Planetária, Fitoterapia e Antropologia Médica.

Outro aspecto destacado é o enfoque baseado em evidências, com forte ênfase em prevenção quaternária. Os princípios de advocacia médica, primum non nocere e os conceitos de overdiagnosis, overtreatment e disease mongering, aparecem constantemente a partir da prática dos profissionais, que moram no mesmo local e estão em contato contínuo com seus pacientes. Esta realidade também facilita a visualização de resultados concretos do trabalho na comunidade, 
o que nem sempre é fácil de obter em estágios com alta rotação e atendimento pontual de casos, como nas emergências, internações e pronto atendimentos, bem como nas especialidades focadas em episódios de doença.

O engajamento comunitário dos estudantes com as "intervenções" de educação em saúde é outro ponto positivo, porque reforça o laço com a comunidade ao oferecer um produto a mais além dos atendimentos individuais e familiares. Estas atividades, que têm ótima avaliação por parte das comunidades e equipes, servem também para revisar assuntos relevantes na medicina e desenvolver as habilidades de trabalho com grupos de pacientes e de comunicação dos estudantes, cada vez mais importante para o trabalho com a saúde, como citado em experiências semelhantes. ${ }^{(24)}$ Ao deslocar-se para municípios menores também possibilita-se aos estudantes um comparativo a respeito do funcionamento do sistema de saúde no interior, que em alguns aspectos pode ser melhor do que nos das grandes cidades. O alto grau de resolutividade das equipes de saúde da família e a boa organização do sistema são frequentemente citados nas avaliações como pontos altos do estágio. Infelizmente esta resolutividade local nem sempre é acompanhada de alta resolutividade dos casos encaminhados para a rede regional de apoio, um sério problema de nosso sistema de saúde nacional.

Todas as unidades apresentam boa estrutura física com todo material necessário aos atendimentos de Atenção Básica, incluindo os principais procedimentos ambulatoriais. Além disso, apresentam boa climatização e informatização, o que ainda não ocorre em todas as unidades de municípios maiores de nossa região.

Os principais desafios enfrentados neste período foram em relação às trocas políticas no poder municipal, que frequentemente geram repetições das negociações com a Universidade. Embora o apoio político tenha sido essencial para o início dos convênios, em nossa realidade, infelizmente, nem sempre são evidentes aos gestores as vantagens na relação com o meio acadêmico e muito menos o papel vital que os serviços de saúde têm para a formação de recursos humanos. Isso gerou diversas vezes dificuldades na renovação dos convênios e na autorização para estágio dos estudantes. O apoio da Universidade foi vital nestes momentos, inclusive com a vinda de coordenadores aos municípios e a elaboração conjunta de ações que potencializam os benefícios às comunidades, como apoio à educação permanente e participação em feiras de saúde.

Outro desafio vem de como lidar com as diferentes percepções de pessoas da comunidade em relação às características de atendimento por médicos em formação, em especial quanto ao tempo de consulta, que pode ser um pouco mais longo. A eventual rotação de membros das equipes de saúde pode contribuir para um pior desfecho de ensino para os estudantes, embora não ocorra com alta frequência nos municípios parceiros.

Ao longo destes anos, os estudantes de Medicina envolveram-se em diversos momentos da comunidade, como participação em conferências de saúde, no planejamento e avaliação de políticas, em pesquisas, contato com o programa municipal de uso de plantas medicinais, e educação em saúde em escolas, fábricas e creches.

Outro ponto positivo da integração ensinoserviço é o estímulo constante ao aperfeiçoamento técnico das equipes, provocado pelas diferentes visões e necessidades dos alunos que vivenciam o estágio. Como reflexo desse aspecto, temos a conclusão do mestrado realizado por dois professores e um doutorado em andamento. A integração dos alunos com a equipe enriquece as relações de trabalho, ponto fundamental para o desenvolvimento de uma atenção primária de qualidade, e isso se reflete em bons indicadores de saúde nas comunidades.

\section{Conclusão}

O estágio rural em Saúde da Família da UCS tem sido um importante espaço de diversificação de cenário para educação médica, reforçando o papel da Atenção Primária à Saúde e a importância da medicina de família e comunidade. Esta experiência de formação médica também parece 
facilitar a aproximação do meio acadêmico com a comunidade regional reforçando a responsabilidade social da Universidade de Caxias do Sul (UCS). Relatos das experiências de educação médica em ambientes diversos em nosso País são necessários, bem como estudos com metodologias avaliativas para embasar estratégias e políticas de formação médica e potencialmente melhorar a equidade na distribuição de recursos humanos em saúde.

\section{Conflito de interesse}

Este trabalho é autofinanciado pelos autores e é resultado parcial de tese de doutorado do primeiro autor. Os autores declaram não haver conflito de interesse, além de estarem ligados como preceptores ao estágio rural do internato médico em MFC e à UCS, conforme apresentado.

\section{Referências}

1 World Health Organization (WHO). Increasing access to health workers in remote and rural areas through improved retention: global policy recommendations [Internet]. Geneva (CHE): World Health Organization (WHO); 2010. [cited 2020 Jul 17]. Available from: https://apps.who.int/iris/bitstream/ handle/10665/44369/9789241564014_eng.pdf;j sessionid $=$ C257FEC91F3934AF1848A65797E $\mathrm{AD} 8 \mathrm{E} 0$ ?sequence $=1$

2 Scheil-Adlung X, editor. Global evidence on inequities in rural health protection: new data on rural deficits in health coverage for 174 countries. [Internet]. Geneva (CHE): International Labour Organization; 2015. [cited 2020 Jul 17]. Available from: https://www.social-protection. org/gimi/gess/RessourcePDF.action?ressource. ressourceId $=51297$

3 Targa LV. Wynn-Jones J, Howe A, Anderson MIP, Lopes JMC, Lermen N Jr, et al. Declaração de Gramado pela Saúde Rural nos países em desenvolvimento. Rev Bras Med Fam Comunidade [Internet]; 2014 jul [citado 2020 jul 17];9(32): 292-4. Disponível em: https://www. rbmfc.org.br/rbmfc/article/view/982/644
4 Wilson N, Couper I, Vries E, Reid S, Fish T, Marais B. A critical review of interventions to redress the inequitable distribution of healthcare professionals to rural and remote areas. Rural Remote Health. [Internet]; 2009 [citado 2020 jul 17];9: 1060. Available from: http://www.rrh.org.au/articles/subviewnew. asp?ArticleID $=1060$

5 Guilbault RWR, Vinson JA. Clinical medical education in rural and underserved areas and eventual practice outcomes: a systematic review and meta-analysis. Educ Health. [Internet]; 2017 [cited 2020 Jul 17];30(2): 146-55. Available from: http://www.educationforhealth. net/article.asp?issn=1357-6283;year=2017; vol ume $=30$; issue $=2$; spage $=146$; epage $=155$; aulast $=$ Raymond

6 O'Sullivan BG, McGrail MR, Russell D, Chambers H, Major L. A review of characteristics and outcomes of Australia's undergraduate medical education rural immersion programs. Hum Resour Health. [Internet]; 2018 [cited $2020 \mathrm{Jul}$ 17];16(1): 8. Available from: https:// human-resources-health.biomedcentral.com/ articles/10.1186/s12960-018-0271-2

7 Villiers M, van Schalkwyk S, Blitz J, Couper I, Moodley K, Talib Z, et al. Decentralised training for medical students: a scoping review. BMC Med Educ. [Internet]; 2017 [cited 2020 Jul 17];17: 196. Available from: https://www. ncbi.nlm.nih.gov/pmc/articles/PMC5680751/ pdf/12909_2017_Article_1050.pdf

8 McLean R, Pallant J, Cunningham C, DeWitt D.A multi-university evaluation of the rural clinical school experience of Australian medical students. Rural Remote Health. [Internet]; 2010 [cited 2020 Jul 17];10(3): 1492. Available from: www.rrh.org.au/journal/article/1492

9 Taveira, MGMM. Clínica ampliada: as oportunidades de vivência discente no estágio rural em Arapiraca [dissertação]. Maceió, Alagoas: Universidade Federal de Alagoas; 2014.

10 Silveira R, Pinheiro R. Internato rural na Amazônia: aspectos históricos, contexto atual e principais desafios. Hist Cienc Saúde-Manguinhos [Internet]. 2017 abr 
[citado 2020 jun 29];24(2): 371-90. Disponível em: http://www.scielo.br/scielo. php? script $=$ sci_arttext \&pid=S $0104-$ 59702017000200371\&lng=pt. doi: https://doi. org/10.1590/s0104-59702017000200004

11 Brasil. Ministério da Educação. Resolução n ${ }^{\circ}$ 3, de 20 de junho de 2014. Institui Diretrizes Curriculares Nacionais do Curso de Graduação em Medicina e dá outras providências. Diário Oficial [da] República Federativa do Brasil. 2014 jun. 23; Seção 1, p. 8.

12 Ministério da Saúde (BR). Política Nacional de Saúde Integral das Populações do Campo e da Floresta [Internet]. Brasília: Ministério da Saúde, 2013. [citado 2020 jul 17]. Disponível em: http://bvsms.saude.gov.br/bvs/publicacoes/ politica_nacional_saude_populacoes_campo. pdf

13 Balint M. O médico, seu paciente e a doença. Rio de Janeiro: Atheneu; 1988.

14 Targa LV, Schwalm F, Hoblik M. Estágio rural potencializa interiorização do médico de família e comunidade. Anais do $10^{\circ}$ Congresso Brasileiro de Medicina de Família e Comunidade; 2009. Florianópolis: SBMFC, 2009.

15 Brasil. Lei $n^{\circ} 12.871$ de 22 de outubro de 2013. Institui o programa mais médicos, altera as leis $\mathrm{n}^{\mathrm{o}} 8.745$, de 9 de dezembro de 1993 , e n ${ }^{\circ} 6.932$, de 7 de julho de 1981, e dá outras providências. [Internet]. 2013. [citado 2020 jul 3]. Disponível em: https://legislacao.presidencia.gov.br/atos/ ?tipo $=$ LEI\&numero $=12871 \&$ ano $=2013 \&$ ato $=$ bbao3ZU50MVpWTde7

16 Instituto Brasileiro de Geografia e Estatística (IBGE). Classificação e caracterização dos espaços rurais e urbanos do Brasil: uma primeira aproximação. Rio de Janeiro: IBGE; 2017.

17 Silveira R, Pinheiro R. Sobre o rural, a interiorização na saúde e a formação médica: concepções e ações com o destaque para o contexto da Amazônia legal. In: Pinheiro R, Lopes T, Müller JS Neto. Construção social da demanda por cuidado: revisitando o direito à saúde, o trabalho em equipe, os espaços públicos e a participação. [Internet]. Rio de Janeiro: Cepesc; 2013. p. 147-172. [citado 2020 jul 17]. Disponível em: https://lappis. org.br/site/construcao-social-da-demandapor-cuidado-revisitando-o-direito-saude-otrabalho-em-equipe-os-espacos-publicos-eparticipacao/4624

18 Guimarães EV. O internato rural e a formação do profissional médico para o programa de saúde da família [dissertação]. Belo Horizonte: Universidade Federal de Minas Gerais - UFMG; 2004.

19 Polignano MV, Melo EM, Nascimento LE, Santana AMP, Rodrigues IA Jr. Internato rural da faculdade de medicina da UFMG: 25 anos de integração docente-assistencial. Anais do $2^{\circ}$ Congresso Brasileiro de Extensão Universitária [Internet]; 2004 set 12-15; Belo Horizonte, MG, Brasil. [citado 2020 jul 17]. Disponível em: https://www.ufmg.br/congrext/ Educa/Educa107.pdf

20 Gouveia EAH, Rodrigues LHG, Maggi RS. Internato rural no sertão indígena de Pernambuco: um projeto piloto. Anais CBMFC. [Internet]; 2013 [citado 2020 jul 17];(12): 129. Disponível em: https://cmfc.emnuvens.com.br/ brasileiro/article/view/975

21 Barbosa GL, Amorim RKM. Contribuição do internato rural na formação do Médico. Anais CBMFC. [Internet]; 2013 [citado 2020 jul 17];(12): 1078. Disponível em: https://www. cmfc.org.br/brasileiro/article/view/571

22 Silveira RP. Itinerários formativos do Internato Rural na Amazônia: experiências nas fronteiras de mundo, saberes e práticas e responsabilidade [tese]. Rio de Janeiro: Universidade do Estado do Rio de Janeiro, Instituto de Medicina Social; 2014.

23 Ruiz DG, Farenzena GJ, Haeffner LSB. Internato regional e formação médica: percepção da primeira turma pós-reforma curricular. Rev Bras Educ Méd. [Internet]; 2010 mar [citado 2020 jul 17]; 34(1): 21-7. Disponível em: http://www.scielo.br/scielo.php?script=sci abstract\&pid=S0100-55022010000100004\&ln $\mathrm{g}=\mathrm{pt} \& \mathrm{nrm}=\mathrm{iso} \& \mathrm{tlng}=\mathrm{pt}$ 
24 Silva MJF, Gonçalves BH, Moraes CAC, Salgado AKS, Rodrigues BC, Vallinoto IMVC. A extensão universitária como cenário de prática e de protagonismo do graduando em medicina na atenção primária em saúde na ilha de Marajó, Estado do Pará. Interfaces [Internet]; 2019 jun [citado 2020 jul 17];7(1):501-10. Disponível em: https://periodicos.ufmg.br/ index.php/revistainterfaces/article/view/19083 